\title{
Surface characterization of $\mathrm{Mn}_{x} \mathrm{Ge}_{1-x}$ and $\mathrm{Cr}_{y} \mathrm{Mn}_{x} \mathrm{Ge}_{1-x-y}$ dilute magnetic semiconductors
}

\author{
P. Gambardella \\ ICREA and ICN, Campus Universitat Autonoma de Barcelona, E-08193 Bellaterra, Spain \\ and Institut de Physique des Nanostructures, Ecole Polytechnique Fédérale de Lausanne, CH-1015 Lausanne, Switzerland \\ L. Claude, S. Rusponi, K. J. Franke, and H. Brune \\ Institut de Physique des Nanostructures, Ecole Polytechnique Fédérale de Lausanne, CH-1015 Lausanne, Switzerland \\ J. Raabe and F. Nolting \\ Swiss Light Source, Paul Scherrer Institut, CH-5232 Villigen PSI, Switzerland \\ P. Bencok \\ European Synchrotron Radiation Facility, Boîte Postale 200, F-38043 Grenoble, France
}

A. T. Hanbicki and B. T. Jonker

Naval Research Laboratory, Washington, DC 20375, USA

C. Grazioli, M. Veronese, and C. Carbone

Istituto di Struttura della Materia, Consiglio Nazionale delle Ricerche, Area Science Park, I-34012 Trieste, Italy

(Received 4 December 2006; revised manuscript received 1 February 2007; published 28 March 2007)

\begin{abstract}
We have used x-ray photoemission electron microscopy (XPEEM) and x-ray absorption spectroscopy (XAS) to characterize $\mathrm{Mn}_{x} \mathrm{Ge}_{1-x}$ and $\mathrm{Cr}_{y} \mathrm{Mn}_{x} \mathrm{Ge}_{1-x-y}$ films grown by molecular beam epitaxy. The surface layers of the as-grown films probed by XPEEM present segregation of Mn-rich phases. XAS using both total electron yield and fluorescence yield detection shows that the films are heavily oxidized after exposure to air. Etching in $\mathrm{HF}$ and $\mathrm{HCl}$ can be used to reduce oxidation, but inhomogeneities in the surface composition might not be completely eliminated depending on the Mn concentration. X-ray magnetic circular dichroism (XMCD) measurements reveal that neither the etched nor the as-grown films present remanent ferromagnetic behavior down to a temperature of $5 \mathrm{~K}$ within the probing depth of the fluorescence yield $(\sim 20 \mathrm{~nm})$. Mn is paramagnetic in both the oxidized and etched samples, with an increased tendency to order magnetically toward the interior of the films. $\mathrm{Cr}$ in $\mathrm{Cr}_{y} \mathrm{Mn}_{x} \mathrm{Ge}_{1-x-y}$ possesses a paramagnetic moment only in the oxidized form. A comparison of the XAS line shapes obtained in the present study with those of Mn impurities deposited on Ge and GaAs surfaces demonstrates that the interpretation of XAS spectra of Mn-doped dilute magnetic semiconductors in the literature is often affected by residual oxidation.
\end{abstract}

DOI: 10.1103/PhysRevB.75.125211 PACS number(s): 75.50.Pp, 75.70.-i, 78.70.Dm, 75.20.Hr

\section{INTRODUCTION}

Substitutional incorporation of transition-metal dopants into nonmagnetic semiconductors such as GaAs (Ref. 1) and Ge (Ref. 2) in nonequilibrium growth conditions leads to ferromagnetic ordering in the doped compounds with Curie temperatures up to $250 \mathrm{~K} .{ }^{3}$ Dilute magnetic semiconductors (DMS) obtained in this way offer exciting opportunities to combine the electronic and optical properties of semiconducting materials with the storage and processing of magnetic information. ${ }^{4,5}$ Although most activity is focused on group III-Mn-V materials, there is steadily increasing interest in Ge-based DMS, driven by their potential integration in standard Si semiconductor technology.,6-13

Several growth techniques have been employed to produce MnGe compounds, including low-temperature molecular beam epitaxy (MBE), ${ }^{2,6-9}$ furnace melting, ${ }^{14,15}$ and implantation of high-energy $\mathrm{Mn}^{2+}$ ions into single crystal Ge wafers. ${ }^{16-18}$ The investigation of the magnetic properties of MnGe compounds, however, reveals very significant differences depending on the growth method. $\mathrm{Mn}_{x} \mathrm{Ge}_{1-x}$ films with $x=2.5-5 \%$ grown by MBE between $50{ }^{\circ} \mathrm{C}$ and $85^{\circ} \mathrm{C}$ yield an extrapolated Curie temperature of about $115 \mathrm{~K}$ from a Curie-Weiss fit of the susceptibility and ferromagnetic remanence below $20 \mathrm{~K}, 2,7$ while films grown at $160^{\circ} \mathrm{C}$ present hysteretic behavior up to $250 \mathrm{~K},{ }^{19}$ and $\mathrm{Mn}_{x} \mathrm{Ge}_{1-x}$ single crystals obtained from melted powders are antiferromagnetic below $150 \mathrm{~K}$ and ferromagnetic from 150 to $285 \mathrm{~K} .{ }^{14,15}$ These apparently conflicting observations can be rationalized in terms of coexisting chemically inhomogeneous $\mathrm{Mn}$ phases $^{7,8,15,16,18}$ and by the variety of Mn-rich precipitates that exhibit ferromagnetism up to room temperature, such as $\mathrm{Mn}_{11} \mathrm{Ge}_{8}$ (Ref. 20) and $\mathrm{Mn}_{5} \mathrm{Ge}_{3} \cdot{ }^{21}$ According to transmission electron microscopy cross-sectional analysis and x-ray diffraction data, the formation of MnGe precipitates is inhibited only in epitaxial thin films grown below $85^{\circ} \mathrm{C}$. 2,7 Such substitutional $\mathrm{Mn}_{x} \mathrm{Ge}_{1-x}$ DMS present hysteretic behavior at low temperature $(T \leqslant 20 \mathrm{~K})$, and evidence of short-range ferromagnetic order up to about $115 \mathrm{~K}, 2,7$ which has been attributed to the formation of bound magnetic polarons. ${ }^{7,22}$ Gated extraordinary Hall effect measurements have proven that ferromagnetic order in these systems is enhanced or suppressed depending on hole injection or depletion, respectively, as expected for Mn-doped DMS. ${ }^{2}$ 
The aim of the present study is to characterize the surface composition and magnetic behavior of $\mathrm{Mn}_{x} \mathrm{Ge}_{1-x}$ and $\mathrm{Cr}_{y} \mathrm{Mn}_{x} \mathrm{Ge}_{1-x-y}$ DMS films grown by low temperature MBE, which we show to differ from the bulk owing to segregation of the dopants, surface contamination, and interface effects. The interest in this type of investigation is twofold. On the one hand, it is important to establish the interface properties of DMS due to the dependence of spin transport on the nature and quality of DMS surfaces ${ }^{23}$ and their anticipated integration in layered semiconductor heterostructure devices as magnetic elements and spin polarizers. ${ }^{4,5}$ On the other hand, as X-ray absorption spectroscopy (XAS) is one of the main tools employed to study the dilution and valence configuration of Mn in DMS, ${ }^{15,17,24-30}$ we wish to emphasize the need of XAS data representative of pure and homogeneous DMS, i.e., with a minimal amount of surface contaminants and phase segregation. By comparing XAS spectra recorded using total electron yield (TEY) detection with fluorescence yield (FY) spectra and the TEY spectra of Mn impurities deposited on $\mathrm{Ge}$ and GaAs surfaces, we show that the ground state of substitutional $\mathrm{Mn}$ in $\mathrm{Ge}$, as also in $\mathrm{GaAs},{ }^{29,30}$ has a hybridized character. Well-defined multiplets in the $L_{2,3}$ XAS spectra of $\mathrm{Mn}$, indicative of a localized $d^{5}$ ground state and observed both in $\mathrm{Mn}_{x} \mathrm{Ge}_{1-x}$ (Refs. 15 and 17) and $\mathrm{Ga}_{1-x} \mathrm{Mn}_{x} \mathrm{As},{ }^{24-26,28}$ originate from oxidation of the Mn atoms in the surface layers within the XAS probing range.

\section{EXPERIMENTAL TECHNIQUES}

$\mathrm{Mn}_{x} \mathrm{Ge}_{1-x}$ and $\mathrm{Cr}_{y} \mathrm{Mn}_{x} \mathrm{Ge}_{1-x-y}$ films with typical thickness of $1000 \AA$ were grown by MBE on $\operatorname{GaAs}(001)$ substrates at $T=70{ }^{\circ} \mathrm{C}$ and left uncapped. Their bulk structural and magnetic properties are described in Refs. 2 and 6. In the present study we investigated films with composition $\mathrm{Mn}_{0.018} \mathrm{Ge}_{0.982}$, $\mathrm{Mn}_{0.025} \mathrm{Ge}_{0.975}$, and $\mathrm{Cr}_{0.022} \mathrm{Mn}_{0.025} \mathrm{Ge}_{0.953}$, the last two corresponding to samples Nos. 515A and 516 in Ref. 6. The Mn concentrations were determined by small-spot transmission electron microscopy analysis of the interior of the film. ${ }^{2}$ The magnetization measured by superconducting quantum interference device (SQUID) prior to the XAS measurements as a function of temperature and applied field was consistent with previously reported data, ${ }^{2,6}$ displaying remanent ferromagnetism at $5 \mathrm{~K}$, as shown in Fig. 1.

X-ray photoemission electron microscopy (XPEEM) was performed at the SIM beamline of the Swiss Light Source. XPEEM images were recorded at room temperature as a function of photon energy over the $L_{2,3}$ edges of $\mathrm{Mn}, \mathrm{Cr}$, and $\mathrm{Ge}$, and the $K$ edge of $\mathrm{O}$ with a field of view of $50 \mu \mathrm{m}$. Elemental contrast was obtained by dividing pixel-by-pixel the intensity of two consecutive images recorded at pre-edge and on-edge energies for each element. Images recorded with left or right circular polarization showed no detectable difference due to the room temperature nonmagnetic response of the samples. XAS and x-ray magnetic circular dichroism (XMCD) spectra were measured as a function of $T$ and applied field B at beamline ID08 of the European Synchrotron Radiation Facility using simultaneous TEY and FY detection. The TEY signal was given by the sample drain current measured by an electrometer, while the FY was measured by

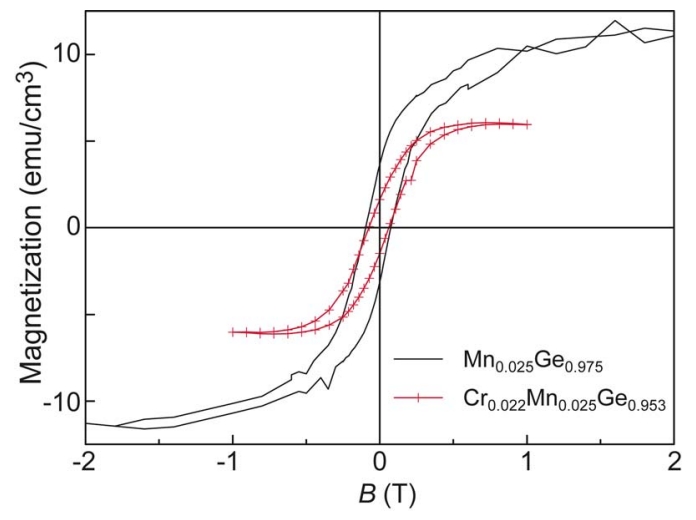

FIG. 1. (Color online) Field-dependent in-plane magnetization of $\mathrm{Mn}_{0.025} \mathrm{Ge}_{0.975}$ (solid line) and $\mathrm{Cr}_{0.022} \mathrm{Mn}_{0.025} \mathrm{Ge}_{0.953}$ (crosses) measured by SQUID at $T=5 \mathrm{~K}$.

means of a Si photodiode detector. The angle of incidence of the X-ray beam was set to $\theta=45^{\circ}$ with respect to the surface normal and the XMCD was recorded by reversing the alignment of the photon helicity relative to B. Films from the same wafer were measured as-grown and after etching in dilute solutions (1:10) of $\mathrm{HF}: \mathrm{H}_{2} \mathrm{O}$ and $\mathrm{HCl}: \mathrm{H}_{2} \mathrm{O}$ for $30 \mathrm{~s}$ each. Here HF was used to selectively etch Ge oxide, while $\mathrm{HCl}$ served to remove segregated $\mathrm{Mn}$ and $\mathrm{Mn}$ oxide, as will be shown below. The etched samples were rinsed in deionized $\mathrm{H}_{2} \mathrm{O}$ and exposed to air for up to 10 minutes before introduction into the ultrahigh-vacuum environment.

\section{RESULTS AND DISCUSSION}

\section{A. X-ray photoemission electron microscopy}

Figure 2 shows a series of XPEEM images of the asgrown $\mathrm{Cr}_{0.022} \mathrm{Mn}_{0.025} \mathrm{Ge}_{0.953}$ sample with $\mathrm{Mn}, \mathrm{Cr}, \mathrm{O}$, and $\mathrm{Ge}$ contrast. Bright and dark regions correspond to relative high or low concentration of the respective elements in the topmost surface layers. As for XAS in the TEY mode, the probing depth of XPEEM is given by the mean free path of the secondary photoelectrons that escape the sample's surface (typically in the range of $2 \mathrm{~nm}$, although variations are observed from element to element). The XAS spectra to the left of the images represent the average intensity of the photoelectrons emitted from the rectangular (solid line) and circular (dashed line) regions indicated on the top image. The analysis of the images reveals at first glance that the film surface is not homogeneous owing to the segregation of $\mathrm{Mn}$ rich phases with poor $\mathrm{Cr}$ and $\mathrm{Ge}$ content. A close inspection of the images recorded at the $\mathrm{O} K$ edge shows that $\mathrm{O}$ is more abundant in such phases. These observations are confirmed by the difference in the relative intensity of the XAS spectra measured in correspondence of the Mn-rich regions (dashed lines) with respect to the homogeneous parts of the surface (solid lines). The elemental spectra are normalized to the pre-edge intensity for ease of comparison.

No Cr-rich phases have been observed within the XPEEM resolution. The intense O XAS peaks further reveal that the whole film surface is heavily oxidized. In fact, the sharp multiplet structures observed in the Mn and Cr XAS in Fig. 

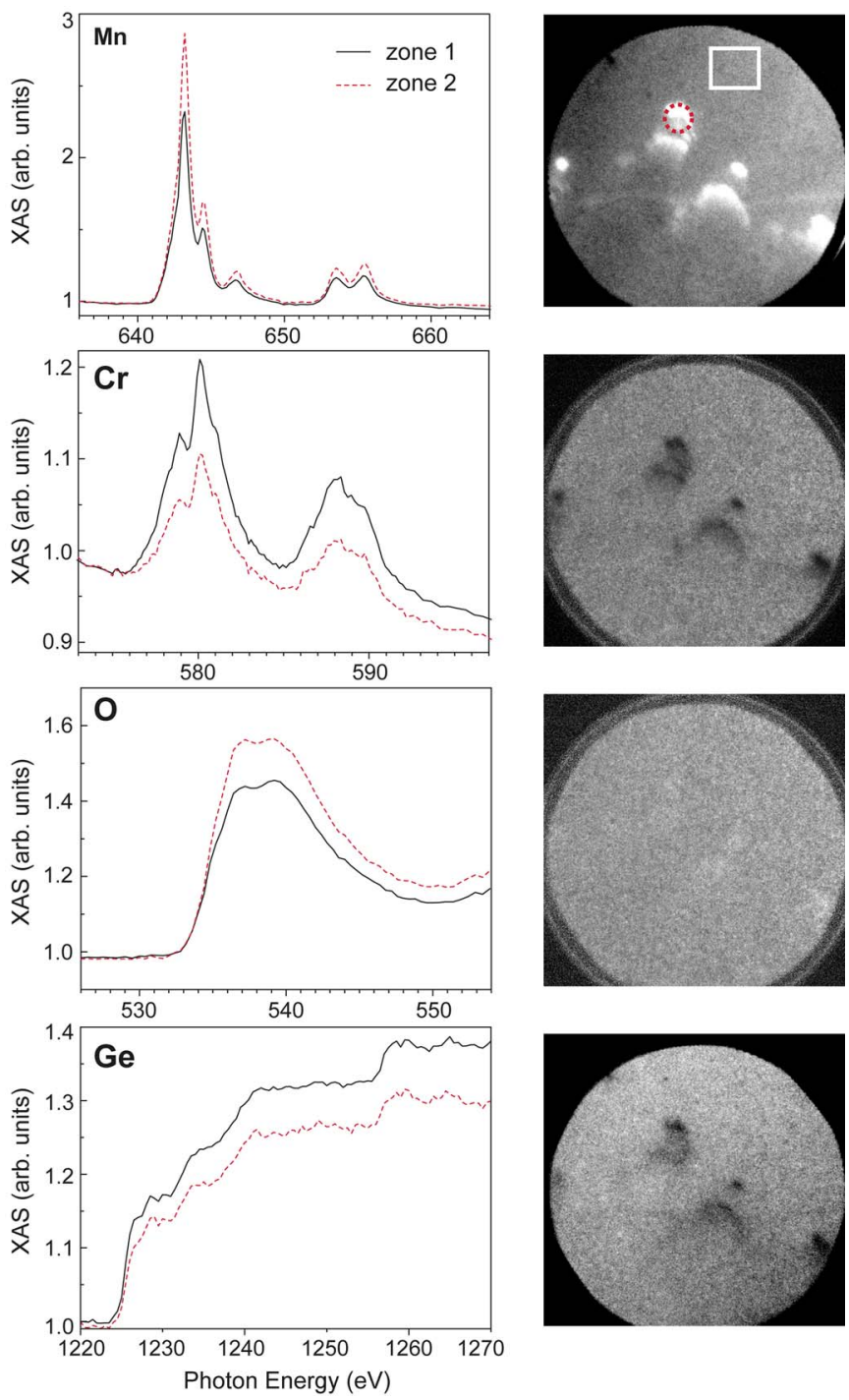

FIG. 2. (Color online) X-ray absorption spectra and XPEEM images $\left(50 \times 50 \mu \mathrm{m}^{2}\right)$ of as-grown $\mathrm{Cr}_{0.022} \mathrm{Mn}_{0.025} \mathrm{Ge}_{0.953}$ recorded at the $\mathrm{Mn} L_{2,3}$ edge, $\mathrm{Cr} L_{2,3}$ edge, $\mathrm{O} K$ edge, and Ge $L_{2,3}$ edge. Light (dark) regions in the images correspond to high (low) concentration of a given element. The solid (dashed) line spectra represent the pixel-averaged intensity of the regions delimited by the rectangle (circle).

2 are due to electron localization and are typical of $\mathrm{MnO}$ (Refs. 31 and 32) and $\mathrm{CrO}_{2}$ (Refs. 33 and 34) compounds. The Mn $d$-state in the oxide compound, in particular, is very close to the atomic $d^{5}$ ground state by virtue of the stability of the ${ }^{6} \mathrm{~S}$ term of a half-filled $d$-shell (see Sec. III B). ${ }^{35,36}$ Apart from the absence of Cr, XPEEM images of the asgrown $\mathrm{Mn}_{x} \mathrm{Ge}_{1-x}$ films reveal analogue features to those described above, namely the presence of $\mathrm{Mn}$ - and O-rich phases with $\mu \mathrm{m}$ size dimensions and significant oxidation of the film surface.

After etching in $\mathrm{HF}$ and $\mathrm{HCl}$, the $\mathrm{Cr}_{y} \mathrm{Mn}_{x} \mathrm{Ge}_{1-x-y}$ and $\mathrm{Mn}_{x} \mathrm{Ge}_{1-x}$ samples present a more chemically homogeneous surface, and oxidation is considerably reduced. Figure 3 shows XPEEM contrast and XAS spectra representative of the homogeneous surface of etched $\mathrm{Mn}_{0.018} \mathrm{Ge}_{0.982}$. The $\mathrm{Mn}$
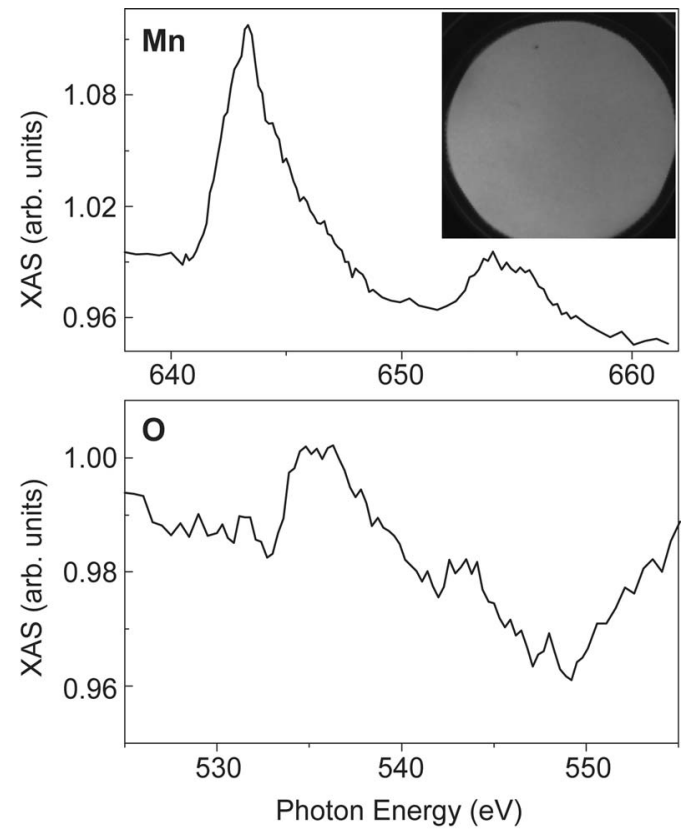

FIG. 3. Representative x-ray absorption spectra of etched $\mathrm{Mn}_{0.018} \mathrm{Ge}_{0.982}$ recorded at the $\mathrm{Mn} L_{2,3}$ edge and $\mathrm{O} K$ edge. The inset shows uniform Mn contrast in a $50 \times 50 \mu \mathrm{m}^{2}$ XPEEM image.

line shape presents no discernible multiplet structure in this case, in agreement with the low $\mathrm{O}$ contamination evidenced by the O XAS. On $\mathrm{Mn}_{0.025} \mathrm{Ge}_{0.975}$ and $\mathrm{Cr}_{0.022} \mathrm{Mn}_{0.025} \mathrm{Ge}_{0.953}$, however, by scanning the sample surface over $\mathrm{mm}$ regions with a $50 \mu \mathrm{m}$ field of view, we occasionally observe residual inhomogeneities that are not present on $\mathrm{Mn}_{0.018} \mathrm{Ge}_{0.982}$, possibly due to the larger concentration of dopants and increased segregation in the former compounds. One such case is shown in Fig. 4. The gray contrast area is typical of etched $\mathrm{Cr}_{0.022} \mathrm{Mn}_{0.025} \mathrm{Ge}_{0.953}$ with uniform $\mathrm{Mn}, \mathrm{Cr}$, and $\mathrm{Ge}$ composition, and reduced oxidation (solid line XAS spectra). Two Mn-rich inclusions are shown, which appear to be composed of $\mathrm{MnO}$ with negligible $\mathrm{Cr}$ and Ge content (dashed line XAS spectra). We note that the Mn line shape corresponding to the oxidized region is practically identical to that of the asgrown samples (Fig. 2), while the multiplet features are broadened and hardly distinguishable in the spectra corresponding to the uniform region. As the TEY peak-tobackground ratio of the $L_{3} \mathrm{Mn}$ edge decreases by $75 \%$ in etched $\mathrm{Cr}_{0.022} \mathrm{Mn}_{0.025} \mathrm{Ge}_{0.953}$, while the FY intensity stays almost constant (not shown), there is evidence that acid exposure reduces the surface concentration of $\mathrm{Mn}$ relative to the interior of the film.

\section{B. Total electron yield and fluorescence yield $x$-ray absorption spectroscopy}

Further insight into the valence state of Mn in Ge-based DMS comes from the comparison of XAS spectra recorded in the TEY and FY mode. In this section, the XAS is integrated over a $0.1 \times 1 \mathrm{~mm}^{2}$ region with no spatial resolution. Due to the larger escape depth of photons with respect to electrons, FY spectra contain information about deeper layers compared to TEY and XPEEM [typically up to $20 \mathrm{~nm}$ 

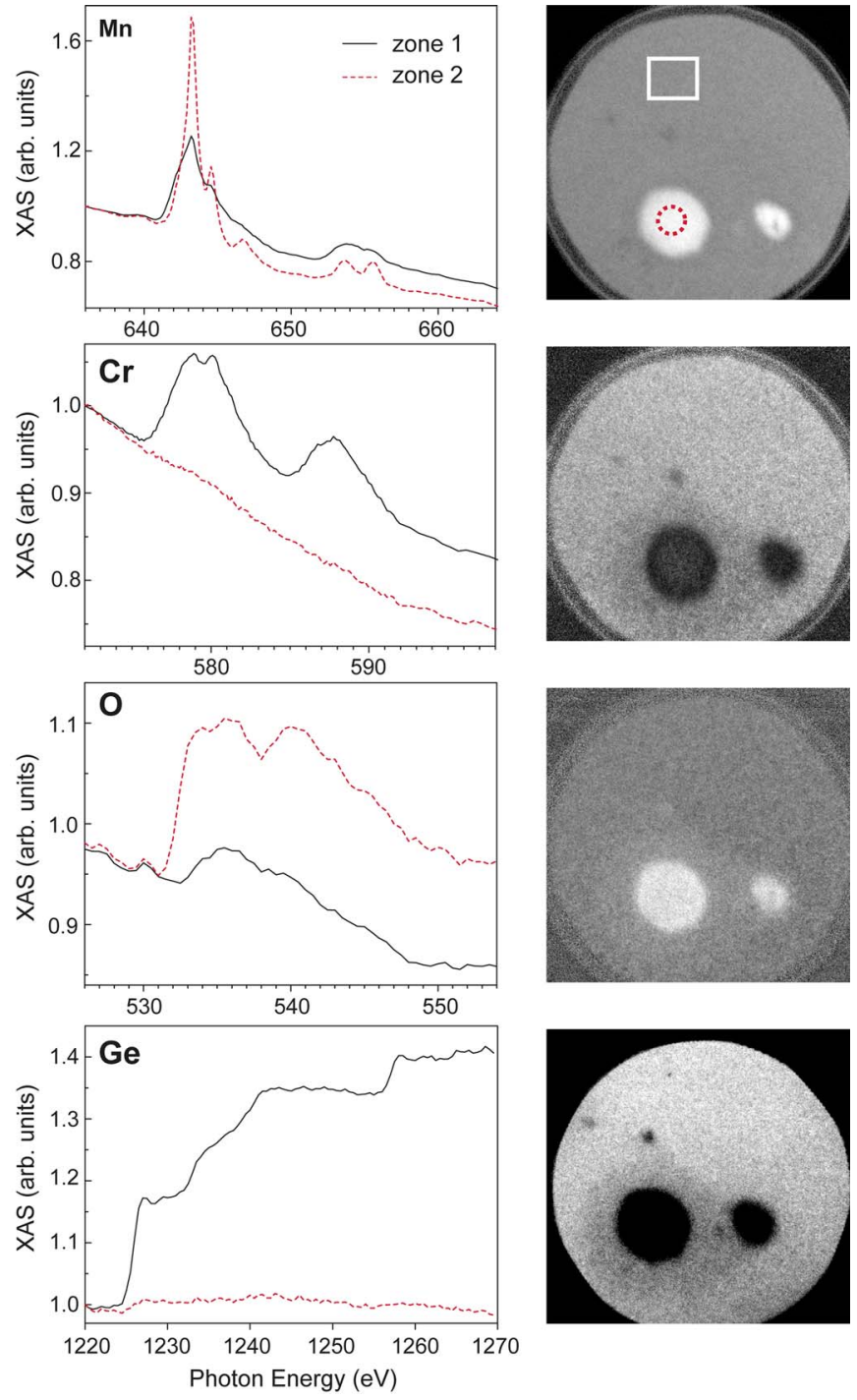

FIG. 4. (Color online) X-ray absorption spectra and XPEEM images $\left(50 \times 50 \mu \mathrm{m}^{2}\right)$ of etched $\mathrm{Cr}_{0.022} \mathrm{Mn}_{0.025} \mathrm{Ge}_{0.953}$ recorded as in Fig. 2.

from the sample surface for FY compared to $2 \mathrm{~nm}$ for TEY (Refs. 37 and 38)]. Figure 5 shows the TEY (a) and FY (b) spectra of as-grown $\mathrm{Mn}_{0.025} \mathrm{Ge}_{0.975}$ and etched $\mathrm{Mn}_{0.018} \mathrm{Ge}_{0.982}$. The spectra represent the sum of the XAS intensity recorded with left $\left(I_{L}\right)$ and right $\left(I_{R}\right)$ circular polarization. We note that no distinction exists between $I_{L}$ and $I_{R}$ in the absence of applied field or magnetic remanence. The TEY data of the as-grown sample reproduce the multiplet features observed in Fig. 2 and in the oxidized regions of Fig. 4. These features are present also in the FY spectrum, showing that Mn oxidation reaches layers $10-20 \mathrm{~nm}$ deep in as-grown $\mathrm{Mn}_{x} \mathrm{Ge}_{1-x}$ exposed to ambient conditions. As expected from the analysis of the preceding section, the multiplet peaks in the TEY of etched $\mathrm{Mn}_{0.018} \mathrm{Ge}_{0.982}$ are broadened due to the reduced oxidation of the surface layers. However, contrary to the sample studied by XPEEM (Fig. 3), oxidation in this sample is not entirely removed. A possible cause is the additional time (oxidation) intervened between XPEEM and XMCD studies, even though the sample's wafer and the etching pro-
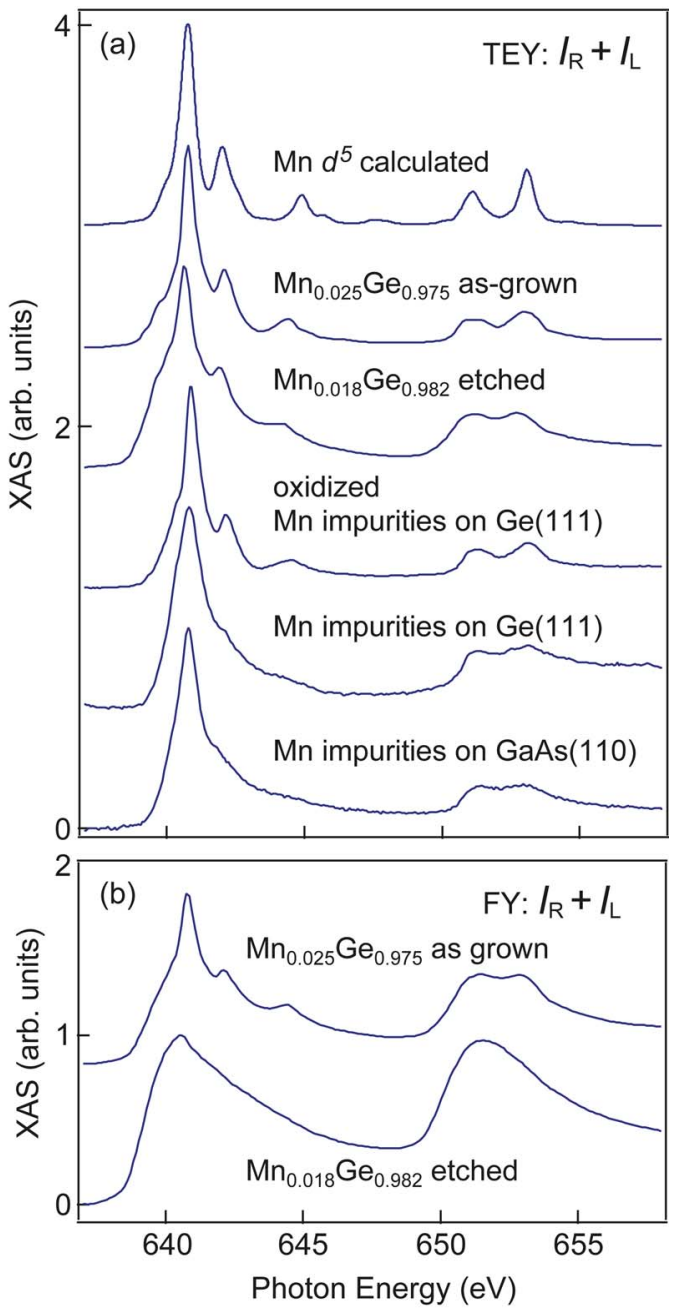

FIG. 5. (Color online) (a) Top to bottom: TEY x-ray absorption spectra of $\mathrm{Mn}$ for the calculated atomic $d^{5}$ configuration, as-grown $\mathrm{Mn}_{0.025} \mathrm{Ge}_{0.975}$, partially etched $\mathrm{Mn}_{0.018} \mathrm{Ge}_{0.982}$, oxidized Mn surface impurities on $\mathrm{Ge}(111)$, individual $\mathrm{Mn}$ impurities on $\mathrm{Ge}(111)$ and on GaAs(110). The substrate absorption background has been subtracted from the impurity spectra; the Mn impurity coverage is 1 $\times 10^{13}$ atoms $/ \mathrm{cm}^{2}$. (b) FY x-ray absorption spectra of as-grown $\mathrm{Mn}_{0.025} \mathrm{Ge}_{0.975}$ and partially etched $\mathrm{Mn}_{0.018} \mathrm{Ge}_{0.982}$. The spectra are shown as measured without correction for self-absorption effects (Ref. 38). All spectra have been normalized to the same height at the $L_{3}$ edge for comparison.

tocol were the same in both cases. In the following we will refer to this case as "partially etched." The absence of multiplet features in the corresponding FY data clearly shows that the inner layers are oxygen free and that the Mn line shape representative of $\mathrm{Mn}_{x} \mathrm{Ge}_{1-x}$ DMS derives from a ground state with a substantial degree of electron delocalization.

In Fig. 5(a) we report for comparison the Mn $L_{2,3}$ XAS calculated for the atomic $d^{5}$ ground state in the absence of crystal field taking into account the $2 p-3 d$ and $3 d-3 d$ Coulomb and exchange interactions, as well as the $2 p$ and $3 d$ spin-orbit interactions, as described in Ref. 36. As mentioned above, such a spectrum is typical of the localized $\mathrm{Mn}^{2+}$ valence state observed in $\mathrm{MnO} .^{31,32,35,36}$ The agreement with 
the XAS measured on the as-grown samples is excellent, showing that the multiplet spectra are due to oxidation rather than being representative of substitutional $\mathrm{Mn}$ in $\mathrm{Ge}$. Indeed, as revealed by the spectra reported in Figs. 3 and 5(b), the line shape of substitutional $\mathrm{Mn}$ is more similar to that observed for metal systems ${ }^{39}$ than to that belonging to a localized configuration with dominant $d^{5}$ character. This is attributed to substantial hybridization between the Mn $3 d$-states with $\mathrm{Ge} 4 p$-states, as shown also by first-principles theoretical calculations. ${ }^{13}$ To lend further support to the line shape assignment we consider the Mn $L_{2,3}$ XAS measured on individual $\mathrm{Mn}$ impurities deposited at low temperature $(5 \mathrm{~K})$ on Ge(111) single crystal surfaces. ${ }^{40}$ The reduced coordination of surface impurities generally favors electron localization, resulting in richly structured XAS spectra. ${ }^{41}$ However, the impurity spectra of $\mathrm{Mn}$ on $\mathrm{Ge}(111)$ present broad and relatively featureless peaks compared to the as-grown $\mathrm{Mn}_{x} \mathrm{Ge}_{1-x}$ films. Intentional oxidation of the surface impurities, on the other hand, leads to the typical $d^{5}$ oxide spectrum.

These conclusions can be generalized to the XAS analysis of $\mathrm{Mn}_{x} \mathrm{Ge}_{1-x}$ compounds reported in the literature, ${ }^{15,17}$ which are evidently affected by oxidation. Analogous deductions were reached for $\mathrm{Ga}_{1-x} \mathrm{Mn}_{x} \mathrm{As}$ compounds. In a recent study, Wu et al. compared TEY and FY XAS spectra of as-grown $\mathrm{Ga}_{1-x} \mathrm{Mn}_{x} \mathrm{As}$, observing that the TEY multiplet structure is absent in FY. ${ }^{42}$ Edmonds et al. showed that the surface of $\mathrm{Ga}_{1-x} \mathrm{Mn}_{x} \mathrm{As}$ films grown by MBE is rich in $\mathrm{Mn}$ and $\mathrm{O}$, which can be removed by etching in $\mathrm{HCl}^{29,30}$ The XAS spectra of the etched compounds are similar to those presented here for Mn impurities deposited on $\operatorname{GaAs}(110)$ and have been interpreted in terms of a mixed $\mathrm{Mn}$ ground state with $16 \% d^{4}, 58 \% d^{5}$, and $26 \% d^{6}$ character. ${ }^{29}$ It is interesting to note that the XMCD, i.e., the magnetic moment per $\mathrm{Mn}$ atom, results to be much larger in $\mathrm{Ga}_{1-x} \mathrm{Mn}_{x} \mathrm{As}$ free of $\mathrm{MnO}$ (Refs. 27, 29, and 30) compared to $\mathrm{Ga}_{1-x} \mathrm{Mn}_{x}$ As where the XAS indicates partial oxidation. ${ }^{24,28}$

Finally, we remark that $\mathrm{Mn}_{x} \mathrm{Ge}_{1-x}$ films present a broader FY linewidth [Fig. 5(b)] compared to $\mathrm{Ga}_{1-x} \mathrm{Mn}_{x} \mathrm{As}$, where the full width at half-maximum of the $\mathrm{Mn} L_{3}$ peak is about $2 \mathrm{eV} \cdot{ }^{27,42}$ This difference might be due to the fact that our FY intensity is not corrected for self-absorption effects, leading to an effective reduction of the peak signal. However, it might also indicate stronger hybridization of $\mathrm{Mn}$ with $\mathrm{Ge}$ relative to GaAs, as well as the presence of multiple Mn site locations within the Ge lattice.

\section{X-ray magnetic circular dichroism}

The magnetic behavior of as-grown and etched $\mathrm{Mn}_{x} \mathrm{Ge}_{1-x}$ and $\mathrm{Cr}_{y} \mathrm{Mn}_{x} \mathrm{Ge}_{1-x-y}$ was studied by XMCD at the $L_{2,3} \mathrm{Mn}$ and $\mathrm{Cr}$ edges. None of the investigated films displayed a remanent XMCD signal in the temperature range 300-5 K, although $\mathrm{Mn}$ atoms possess a paramagnetic moment in all samples. We discuss here the data obtained on partially etched $\mathrm{Mn}_{0.018} \mathrm{Ge}_{0.982}$ to highlight the different magnetization characteristics measured by TEY and FY. Figure 6 shows the XAS recorded for parallel $\left(I_{R}\right.$, dashed line) and antiparallel $\left(I_{L}\right.$, solid line) alignment of the photon helicity with $\mathbf{B}$ $=5 \mathrm{~T}$ at $T=5 \mathrm{~K}$ in the TEY (a) and FY (c) mode. The XMCD
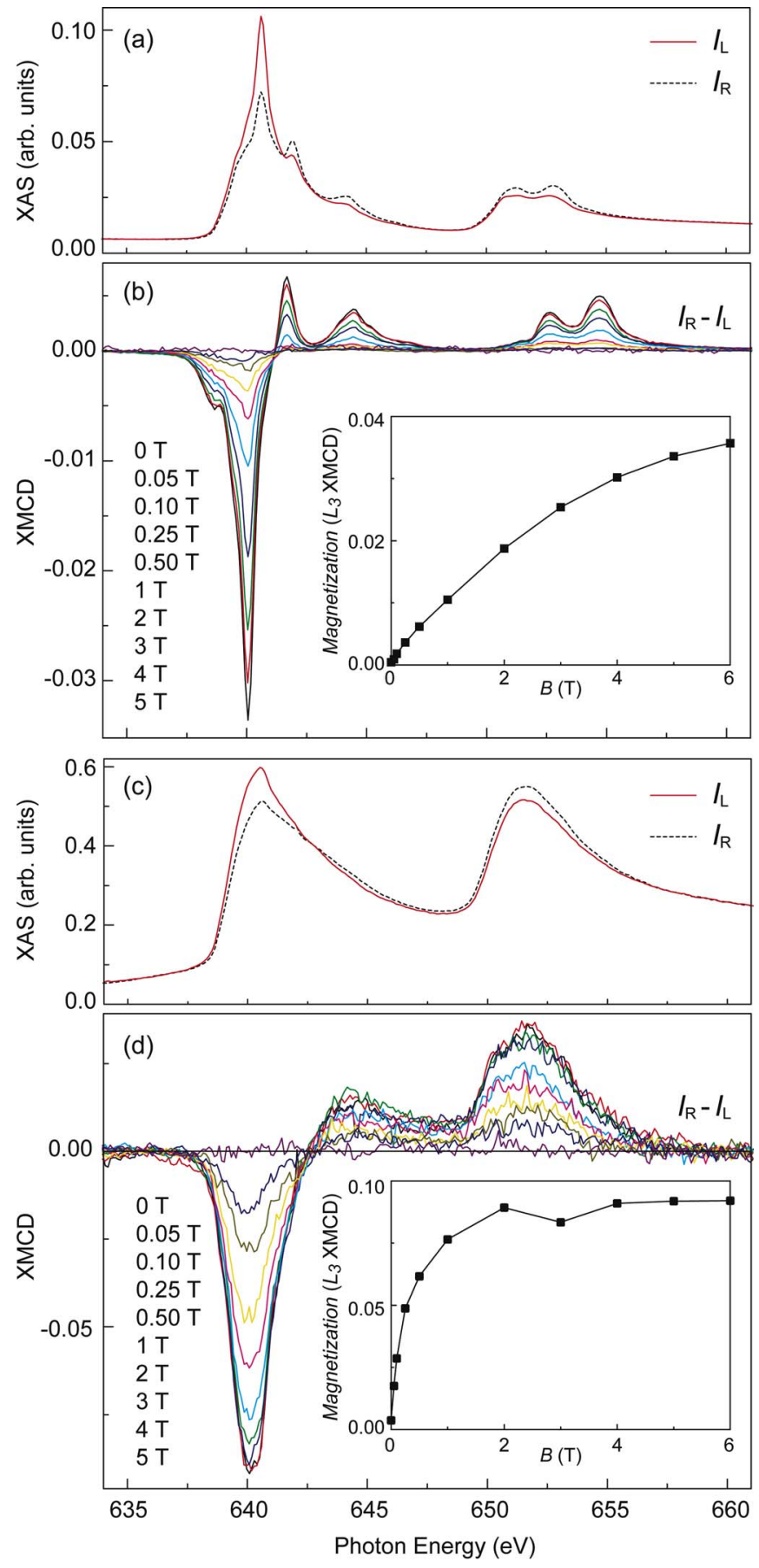

FIG. 6. (Color online) (a) TEY x-ray absorption spectra of partially etched $\mathrm{Mn}_{0.018} \mathrm{Ge}_{0.982}$ recorded for parallel ( $I_{R}$, dashed line) and antiparallel $\left(I_{L}\right.$, solid line) alignment of the photon helicity with applied field $\mathbf{B}=5 \mathrm{~T}$ at $T=5 \mathrm{~K}, \mathrm{Mn} L_{2,3}$ absorption edge. (b) TEY XMCD $\left(I_{R}-I_{L}\right)$ as a function of $\mathbf{B}$ at $T=5 \mathrm{~K}$. The inset shows the Mn magnetization measured by the intensity of the $L_{3}$ peak vs $\mathbf{B}$. XMCD units are given relative to the (arbitrary) XAS intensity. (c) and (d) recorded simultaneously with (a) and (b) in FY.

$\left(I_{R}-I_{L}\right)$ is shown in (b) and (d) for $0 \leqslant \mathbf{B} \leqslant 5 \mathrm{~T}$. The incidence angle of the photon beam was set to $\theta=45^{\circ}$ to probe both in-plane and out-of-plane components of the sample magnetization. The amplitude of the XMCD intensity as a function of $\mathbf{B}$ clearly reveals a paramagnetic behavior. The 


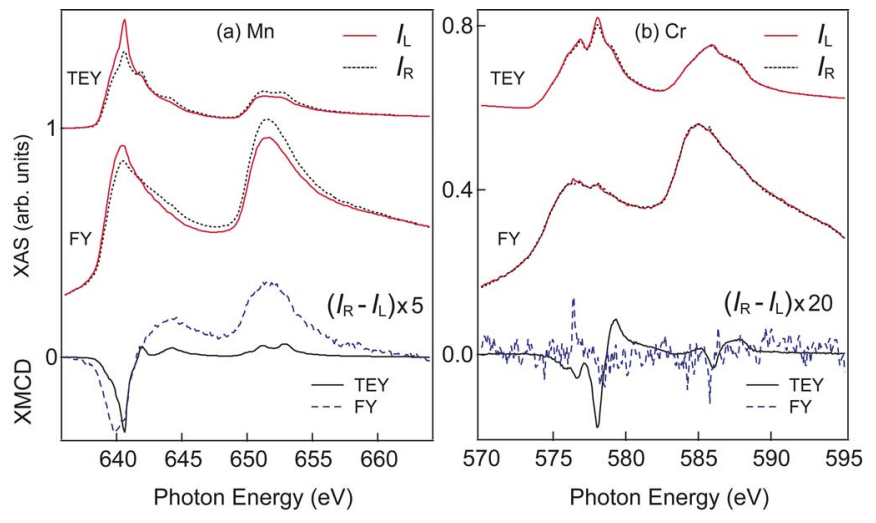

FIG. 7. (Color online) TEY and FY x-ray absorption spectra and XMCD of partially etched $\mathrm{Cr}_{0.022} \mathrm{Mn}_{0.025} \mathrm{Ge}_{0.953}$ recorded with $\mathbf{B}=6 \mathrm{~T}, T=5 \mathrm{~K}$ at the $\mathrm{Mn}(\mathrm{a})$ and $\mathrm{Cr}$ (b) $L_{2,3}$ edge.

absence of magnetic remanence is evident from the flat XMCD observed at $\mathbf{B}=0$. This result is not unexpected for the TEY signal, which is representative of the MnO-rich surface phase, as $\mathrm{MnO}$ is known to be antiferromagnetic with a Néel temperature of $120 \mathrm{~K} .{ }^{43}$ The zero remanence in the FY $\mathrm{XMCD}$, however, is surprising given the bulk hysteretic magnetization reported in Fig. 1 and in Refs. 2 and 6. A conceivable explanation for this effect lies in the dependence of ferromagnetic order on the hole density in $\mathrm{Mn}_{x} \mathrm{Ge}_{1-x},{ }^{2}$ which might be reduced in proximity of the film surface due to hole depletion. ${ }^{44}$ Alternatively, if coalescence of magnetic polarons is considered the mechanism responsible for the onset of hysteretic behavior in $\mathrm{Mn}_{x} \mathrm{Ge}_{1-x}$ below $20 \mathrm{~K},{ }^{7}$ it is possible that missing "links" between polarons on the surface side effectively reduce their magnetic coupling, resulting in a decrease of the temperature at which magnetic ordering occurs. ${ }^{22}$ In agreement with both explanations, the comparison between TEY and FY data reveals a tendency to ferromagnetic coupling that increases towards the film interior. The Mn magnetization recorded as a function of $\mathbf{B}$ [insets of Figs. 6(b) and 6(d)] is steeper for FY compared to TEY, indicating that the Mn susceptibility is larger in the inner layers of the film relative to the surface. Moreover, the FY curve saturates at lower field compared to individual high-spin Mn impurities. ${ }^{40}$ A Brillouin fit of the magnetization with Landé factor $g=2$ gives an effective moment of $5.2 \pm 0.2 \mu_{B}$ for the TEY curve and $64 \pm 6 \mu_{B}$ for the FY curve, which, in the latter case, indicates the presence of shortrange ferromagnetic coupling among $\mathrm{Mn}$ dopants.

Finally, we consider the XMCD of $\mathrm{Cr}_{0.022} \mathrm{Mn}_{0.025} \mathrm{Ge}_{0.953}$, shown separately for $\mathrm{Mn}$ and $\mathrm{Cr}$ in Figs. 7(a) and 7(b). The addition of $\mathrm{Cr}$ was investigated in order to increase the hole density and enhance the magnetization characteristics of $\mathrm{Mn}_{x} \mathrm{Ge}_{1-x}$, but was shown to systematically reduce the Curie temperature and saturation magnetization of the mixed compounds. ${ }^{6}$ In light of these observations, it is interesting to know whether $\mathrm{Cr}$ has a magnetic moment antiferromagnetically coupled to $\mathrm{Mn}$ or no net magnetization. Here we show that $\mathrm{Cr}$ possesses a paramagnetic moment only when oxidized [TEY data in Fig. 7(b)], in which case the sign of the XMCD indicates that it is aligned parallel to the Mn moment. In the FY Cr data, on the other hand, no clear XMCD signal emerges from the noise. Thus $\mathrm{Cr}$ in the interior of the film has no net magnetization, consistently with either a nonmagnetic ground state or strong antiferromagnetic coupling between neighboring $\mathrm{Cr}$ impurities. The observed reduction of the saturation magnetization observed in $\mathrm{Cr}_{y} \mathrm{Mn}_{x} \mathrm{Ge}_{1-x-y}$ in spite of the increased hole density can tentatively be explained by the formation of $\mathrm{MnCr}$ clusters with reduced or no magnetic moment.

\section{CONCLUSIONS}

In summary, we have shown that the surface composition of $\mathrm{Mn}_{x} \mathrm{Ge}_{1-x}$ and $\mathrm{Cr}_{y} \mathrm{Mn}_{x} \mathrm{Ge}_{1-x-y}$ films grown by molecular beam epitaxy is not homogeneous due to segregation of Mnrich phases. The as-grown films present severe oxidation reaching 10-20 nm deep into the films, which can be removed by etching in $\mathrm{HF}$ and $\mathrm{HCl}$ dilute solutions. Contrary to $\mathrm{MnO}$, the Mn XAS line shape representative of oxidationfree $\mathrm{Mn}_{x} \mathrm{Ge}_{1-x}$ has no pronounced multiplet features and reveals delocalization of the $\mathrm{Mn} d$-states due to hybridization with Ge. We did not observe ferromagnetic remanence either by TEY or FY in as-grown or etched samples between 300 and $5 \mathrm{~K}$, implying that the film surface layers have different magnetic properties with respect to the bulk. FY measurements in partially etched $\mathrm{Mn}_{x} \mathrm{Ge}_{1-x}$ show indications of ferromagnetic coupling between $\mathrm{Mn}$ dopants relative to the oxidized topmost layers probed by TEY. $\mathrm{Cr}$ in $\mathrm{Cr}_{y} \mathrm{Mn}_{x} \mathrm{Ge}_{1-x-y}$ has no net magnetization unless it is oxidized, in which case it behaves paramagnetically. These results prove that DMS surface properties such as segregation and contamination, as well as differences in the volume vs interface characteristics of semiconductors might significantly affect their magnetic behavior.

\section{ACKNOWLEDGMENTS}

The authors thank Gilles Retout, ESRF, for technical assistance during the experiment. Part of this work was performed at the Swiss Light Source, Paul Scherrer Institut, Villigen, Switzerland. The work at Naval Research Laboratory was supported by the Office of Naval Research. Financial support from the Swiss National Science Foundation (Grant No. 21-63864) and the Italian Ministero dell' Istruzione, dell'Università e della Ricerca (FIRB project "Nanotecnologie e nanodispositivi optoelettronici, elettronici e spintronici”) is gratefully acknowledged. 
${ }^{1}$ H. Ohno, A. Shen, F. Matsukura, A. Oiwa, A. Endo, S. Katsumoto, and Y. Iye, Appl. Phys. Lett. 69, 363 (1996).

${ }^{2}$ Y. D. Park, A. T. Hanbicki, S. C. Erwin, C. S. Hellberg, J. M. Sullivan, J. E. Mattson, T. F. Ambrose, A. Wilson, G. Spanos, and B. T. Jonker, Science 295, 651 (2002).

${ }^{3}$ A. M. Nazmul, T. Amemiya, Y. Shuto, S. Sugahara, and M. Tanaka, Phys. Rev. Lett. 95, 017201 (2005).

${ }^{4}$ Y. Ohno, D. K. Young, B. Beschoten, F. Matsukura, H. Ohno, and D. D. Awschalom, Nature (London) 402, 790 (1999).

${ }^{5}$ S. A. Wolf, D. D. Awschalom, R. A. Buhrman, J. M. Daughton, S. von Molnár, M. L. Roukes, A. Y. Chtchelkanova, and D. M. Treger, Science 294, 1488 (2001).

${ }^{6}$ G. Kioseoglou, A. T. Hanbicki, C. H. Li, S. C. Erwin, R. Goswami, and B. T. Jonker, Appl. Phys. Lett. 84, 1725 (2004).

${ }^{7}$ A. P. Li, J. Shen, J. R. Thompson, and H. H. Weitering, Appl. Phys. Lett. 86, 152507 (2005).

${ }^{8}$ C. Jaeger, C. Bihler, T. Vallaitis, S. T. B. Goennenwein, M. Opel, R. Gross, and M. S. Brandt, Phys. Rev. B 74, 045330 (2006).

${ }^{9}$ M. Jamet, A. Barski, T. Devillers, V. Poydenot, R. Dujardin, P. Bayle-Guillemaud, J. Rohtman, E. Bellec-Almaric, A. Marty, J. Cibert, R. Mattana, and S. Tatarenko, Nat. Mater. 5, 653 (2006).

${ }^{10}$ F. Tsui, L. He, L. Ma, A. Tkachuk, Y. S. Chu, K. Nakajima, and T. Chikyow, Phys. Rev. Lett. 91, 177203 (2003).

${ }^{11}$ H. Braak, R. R. Gareev, D. E. Bürgler, R. Schreiber, P. Grünberg, and C. M. Schneider, J. Magn. Magn. Mater. 286, 46 (2005).

${ }^{12}$ A. Stroppa, S. Picozzi, A. Continenza, and A. J. Freeman, Phys. Rev. B 68, 155203 (2003).

${ }^{13}$ Y.-J. Zhao, T. Shishidou, and A. J. Freeman, Phys. Rev. Lett. 90, 047204 (2003).

${ }^{14}$ S. Cho, S. Choi, S. C. Hong, Y. Kim, J. B. Ketterson, B.-J. Kim, Y. C. Kim, and J.-H. Jung, Phys. Rev. B 66, 033303 (2002).

${ }^{15}$ J.-S. Kang, G. Kim, S. C. Wi, S. S. Lee, S. Choi, Sunglae Cho, S. W. Han, K. H. Kim, H. J. Song, H. J. Shin, A. Sekiyama, S. Kasai, S. Suga, and B. I. Min, Phys. Rev. Lett. 94, 147202 (2005).

${ }^{16}$ F. D’Orazio, F. Lucari, M. Passacantando, P. Picozzi, S. Santucci, and A. Verna, IEEE Trans. Magn. 38, 2856 (2002).

${ }^{17}$ S. Picozzi, L. Ottaviano, M. Passacantando, G. Profeta, A. Continenza, F. Priolo, M. Kim, and A. J. Freeman, Appl. Phys. Lett. 86, 062501 (2005).

${ }^{18}$ A. Verna, L. Ottaviano, M. Passacantando, S. Santucci, P. Picozzi, F. D’Orazio, F. Lucari, M. De Biase, R. Gunnella, M. Berti, A. Gasparotto, G. Impellizzeri, and F. Priolo, Phys. Rev. B 74, 085204 (2006).

${ }^{19}$ F. D’Orazio, F. Lucari, N. Pinto, L. Morresi, and R. Murri, J. Magn. Magn. Mater. 272, 2006 (2004).

${ }^{20}$ Y. D. Park, A. Wilson, A. T. Hanbicki, J. E. Mattson, T. Ambrose, G. Spanos, and B. T. Jonker, Appl. Phys. Lett. 78, 2739 (2001).

${ }^{21}$ C. Zeng, S. C. Erwin, L. C. Feldman, A. P. Li, R. Jin, Y. Song, J. R. Thompson, and H. H. Weitering, Appl. Phys. Lett. 83, 5002 (2003).

${ }^{22}$ A. Kaminski and S. Das Sarma, Phys. Rev. Lett. 88, 247202
(2002).

${ }^{23}$ J. G. Braden, J. S. Parker, P. Xiong, S. H. Chun, and N. Samarth, Phys. Rev. Lett. 91, 056602 (2003).

${ }^{24}$ H. Ohldag, V. Solinus, F. U. Hillebrecht, J. B. Goedkoop, M. Finazzi, F. Matsukura, and H. Ohno, Appl. Phys. Lett. 76, 2928 (2000).

${ }^{25}$ S. Ueda, S. Imada, T. Muro, Y. Saitoh, S. Suga, F. Matsukura, and H. Ohno, Physica E (Amsterdam) 10, 210 (2001).

${ }^{26}$ Y. Ishiwata, M. Watanabe, R. Eguchi, T. Takeuchi, Y. Harada, A. Chainani, S. Shin, T. Hayashi, Y. Hashimoto, S. Katsumoto, and Y. Iye, Phys. Rev. B 65, 233201 (2002).

${ }^{27}$ Y. L. Soo, G. Kioseoglou, S. Kim, X. Chen, H. Luo, Y. H. Kao, H.-J. Lin, H. H. Hsieh, T. Y. Hou, C. T. Chen, Y. Sasaki, X. Liu, and J. K. Furdyna, Phys. Rev. B 67, 214401 (2003).

${ }^{28}$ D. J. Keavney, D. Wu, J. W. Freeland, E. Johnston-Halperin, D. D. Awschalom, and J. Shi, Phys. Rev. Lett. 91, 187203 (2003).

${ }^{29}$ K. W. Edmonds, N. R. S. Farley, R. P. Campion, C. T. Foxon, B. L. Gallagher, T. K. Johal, G. van der Laan, M. MacKenzie, J. N. Chapman, and E. Arenholz, Appl. Phys. Lett. 84, 4065 (2004).

${ }^{30}$ K. W. Edmonds, N. R. S. Farley, T. K. Johal, G. van der Laan, R. P. Campion, B. L. Gallagher, and C. T. Foxon, Phys. Rev. B 71, 064418 (2005).

${ }^{31}$ Y. Yonamoto, T. Yokoyama, K. Amemiya, D. Matsumura, and T. Ohta, Phys. Rev. B 63, 214406 (2001).

${ }^{32}$ C. Mitra, Z. Hu, P. Raychaudhuri, S. Wirth, S. I. Csiszar, H. H. Hsieh, H.-J. Lin, C. T. Chen, and L. H. Tjeng, Phys. Rev. B 67, 092404 (2003).

${ }^{33}$ D. J. Huang, H.-T. Jeng, C. F. Chang, G. Y. Guo, J. Chen, W. P. Wu, S. C. Chung, S. G. Shyu, C. C. Wu, H.-J. Lin, and C. T. Chen, Phys. Rev. B 66, 174440 (2002).

${ }^{34}$ E. Goering, A. Bayer, S. Gold, G. Schütz, M. Rabe, U. Rüdiger, and G. Güntherodt, Phys. Rev. Lett. 88, 207203 (2002).

${ }^{35}$ F. M. F. de Groot, J. C. Fuggle, B. T. Thole, and G. A. Sawatzky, Phys. Rev. B 42, 5459 (1990).

${ }^{36}$ G. van der Laan and B. T. Thole, Phys. Rev. B 43, 13401 (1991).

${ }^{37}$ S. Eisebitt, T. Böske, J.-E. Rubensson, and W. Eberhardt, Phys. Rev. B 47, 14103 (1993).

${ }^{38}$ R. Nakajima, J. Stöhr, and Y. U. Idzerda, Phys. Rev. B 59, 6421 (1999).

${ }^{39}$ W. L. O’Brien and B. P. Tonner, Phys. Rev. B 50, 2963 (1994).

${ }^{40}$ P. Gambardella, H. Brune, S. S. Dhesi, P. Bencok, S. R. Krishnakumar, S. Gardonio, M. Veronese, C. Grazioli, and C. Carbone, Phys. Rev. B 72, 045337 (2005).

${ }^{41}$ P. Gambardella, S. S. Dhesi, S. Gardonio, C. Grazioli, P. Ohresser, and C. Carbone, Phys. Rev. Lett. 88, 047202 (2002).

${ }^{42}$ D. Wu, D. J. Keavney, R. Wu, E. Johnston-Halperin, D. D. Awschalom, and J. Shi, Phys. Rev. B 71, 153310 (2005).

${ }^{43}$ C. G. Shull, W. A. Strauser, and E. O. Wollan, Phys. Rev. 83, 333 (1951).

${ }^{44}$ The thickness $t$ of the surface depletion layer can be estimated by $t \sim \sqrt{2 \varepsilon \phi_{0} / e N} \sim 10 \mathrm{~nm}$, where $\phi_{0}=0.5 \mathrm{~V}$ represents the band bending and $N=10^{19} \mathrm{~cm}^{-3}$ the carrier density. 